\title{
Análisis de la validez de la Escala de Irritación en una muestra de profesores de Educación Primaria: un estudio exploratorio
}

\author{
Enrique Merino-Tejedor* \\ Universidad de Valladolid, España
}

\begin{abstract}
Resumen: El propósito de este artículo es comprobar la validez de constructo de la versión española de la Escala de Irritación (Irritation Scale) en una muestra de 106 profesores de Educación Primaria. Para ello, los datos obtenidos mediante la Escala de Irritación se contrastaron con otro instrumento que evalúa estrés, el Cuestionario Breve de Burnout adaptado para profesores, esperando obtener correlaciones positivas entre ambos instrumentos. Además, se utilizaron otros dos instrumentos adicionales, la Escala de Autoeficacia General y la Escala de Autorregulación, con la hipótesis de obtener una correlación negativa con la Escala de Irritación. Los resultados obtenidos confirmaron las hipótesis planteadas en este estudio, dado que se obtuvieron correlaciones positivas y significativas entre los resultados de la Escala de Irritación y el Cuestionario Breve de Burnout. Mientras tanto, las correlaciones de la Escala de Irritación con las otras dos escalas fueron negativas y también significativas. Por tanto, los análisis estadísticos corroboran la validación de la Escala de Irritación y su utilidad para la evaluación del estrés entre el profesorado de Educación Primaria.

Palabras clave: Irritación; Burnout; autoeficacia general; autorregulación.
\end{abstract}

\section{Introducción}

La investigación que se presenta en este artículo está centrada en torno al estudio de cuatro conceptos vinculados a la comprensión de la conducta humana y, en particular, al estudio del estrés: la irritación laboral, el síndrome de burnout, la autoeficacia general y la autorregulación. Se trata de un estudio más en el proceso continuo de acumulación de evidencias que es en lo que consiste, en definitiva, la validación de las puntuaciones de un test, en este caso de un instrumento que pretende evaluar la irritación laboral, un concepto que comentaremos a continuación. Concretamente, se pretenden encontrar evidencias empíricas que garanticen la existencia del constructo psicológico irritación, la validez de constructo es una condición necesaria para determinar la eficacia de un instrumento a la hora de medir una variable psicológica. Además, se pretenden encontrar evidencias sobre la validez convergente de la escala, es decir, la correlación entre medidas del mismo constructo utilizando distintos procedimientos de medida, en este caso la correlación entre la irritación y el burnout como indicadores del estrés laboral.

La investigación reciente ha demostrado los efectos negativos del estrés sobre la salud de las personas (Öhman, Bergdahl, Nyberg y Nilsson, 2007; Shirom, 2007). Particularmente, los profesores constituyen un colectivo en el que el tema del estrés ha generado gran número de investigaciones, tal como ponen de manifiesto algunas investigaciones y revisiones teóricas llevadas a cabo en nuestro país (García, 1996; Latorre y Sáez, 2009; Moriana y Herruzo, 2004).

* Dirección para correspondencia [Correspondence address]: Enrique Merino Tejedor. Facultad De Ciencias Sociales, Jurídicas y Comunicación. Casa De La Tierra. Plaza De La Tierra, 3. 40001 Segovia (España). E-mail: enmerino@psi.uva.es
Title: Validity analysis of the Irritation Scale in a sample of Elementary School teachers: an exploratory study.

Abstract: The purpose of the present article is to test the validity of the Spanish version of the Irritation Scale in a sample of 106 Elementary School teachers. Results obtained through the use of the Irritation Scale were compared to those obtained with another instrument, the Brief Burnout Questionnaire adapted for teachers; positive and significant correlations were expected to be found between both instruments. Furthermore, two additional instruments were used, the General Self-Efficacy Scale and the Self-Regulation Scale, with the hypothesis of finding negative correlations between the latter two instruments and the Irritation Scale. Results confirmed the research hypotheses since the Irritation Scale correlated positively and significantly with the Brief Burnout Questionnaire, and negatively and significantly with the other two instruments. Thus, statistical analyses confirm the validation of the Irritation Scale for assessing stress among Elementary School teachers.

Key words: Irritation; burnout; general self-efficacy; self-regulation.

A lo largo de los últimos años, en la actividad docente y en los ambientes educativos ha crecido el estrés entre los docentes. De algún modo, cierto nivel de estrés es necesario y puede ser positivo para realizar una serie de actividades en los escenarios laborales; el problema surge cuando las demandas del escenario laboral sobrepasan los recursos personales que los sujetos disponen, pasando a valorar la situación como una amenaza en lugar de considerarla como un desafío para crecer personalmente, esta situación ha sido planteada por algunos modelos clásicos en el estudio del estrés (Lazarus y Folkman, 1984). Entre los aspectos que suponen una fuente de estrés importante dentro del mundo educativo, podemos destacar los siguientes: la carga cuantitativa del trabajo (número de tareas), la carga cualitativa del trabajo (complejidad de las tareas), la responsabilidad hacia las personas, la responsabilidad hacia las cosas, la ambigüedad de rol, la abundancia de enfrentamientos, las formas de participación, las relaciones con los supervisores y subordinados, así como las propias relaciones con los iguales. Además, existen factores estructurales, como por ejemplo la acumulación de tareas, la saturación de las aulas o la falta de profesionales de apoyo en los centros (Moriana y Herruzo, 2004).

El concepto de irritación (Irritation) se viene desarrollando actualmente en la investigación relacionada con el estrés laboral y fue acuñado originalmente por un equipo de investigación de la universidad alemana de Leipzig (Mohr, 1986). Este nuevo concepto es diferente a otros vinculados al estrés laboral, como por ejemplo el burnout comentado previamente, y está generando gran interés dentro de la comunidad científica de habla hispana. Tal como lo definen los autores en su origen, este concepto hace referencia a un estado de agotamiento psicológico progresivo, el cual no puede paliarse durante los descansos normales. La fatiga puede reducirse durante las pausas normales, algo que no sucede en el caso de la irritación. Por lo tanto, la irritación es más que la fatiga 
psicológica pero sin llegar a tener el rango de una enfermedad psicológica. Algunos estudios longitudinales llevados a cabo en Alemania han demostrado que la irritación es un precedente de algunos deterioros psicológicos adicionales (Dormann y Zapf, 2002). El estado de irritación está vinculado a una experiencia personal de incertidumbre generalizada en contextos laborales e incluye reacciones negativas como la inseguridad, la incomodidad y malestar general.

Desde este punto de vista, el constructo irritación merece una llamada de atención en su contribución al diagnóstico precoz de la tensión psicológica, particularmente en el contexto del análisis de la salud ocupacional. El diagnóstico pre$\mathrm{COZ}$ de este proceso debe tener prioridad para poder prevenir consecuencias costosas para los empleados y para las propias organizaciones. Algunas investigaciones actuales se han centrado en la correcta valoración de este estado psicológico, concretamente con el desarrollo y validación de la Escala de Irritación, un instrumento eficaz para la valoración del estado de irritación de los trabajadores (Mohr, 1986; Mohr, Müller, Rigotti, Aycan y Tschan, 2006). En esta línea, el presente estudio pretende añadir evidencia empírica dentro de un proceso continuo de acumulación de evidencias obtenidas con distintos métodos, de modo que la evidencia acumulada permita interpretar las puntuaciones de la prueba de un modo determinado y apoyar su utilización para la práctica profesional.

En la actualidad, el concepto de irritación va incorporándose progresivamente a la investigación sobre el estrés y se van conociendo más datos sobre su relación con otras variables relevantes en el contexto laboral. Por ejemplo, se han encontrado correlaciones positivas y estadísticamente significativas entre la irritación laboral y variables como la presión del tiempo, el conflicto experimentado en las relaciones entre familia y trabajo y las quejas psicosomáticas (Höge, 2009).

Por otro lado, un aspecto muy estudiado dentro del estrés laboral del profesorado es el síndrome de burnout. El concepto "burnout" con frecuencia ha sido utilizado para referirse a un tipo de estrés generalmente vinculado a las profesiones asistenciales o de servicios, ámbitos en los que han abundado la mayor parte de los estudios realizados en la actualidad (Peltzer, Mashego y Mabeba, 2003; Rohland, Kruse y Rohrer, 2004). Aunque el concepto comenzó a desarrollarse con los escritos de Freudenberger (1974), fue Maslach (1976, 1993) quien le dio el impulso definitivo para cobrar el protagonismo y la importancia que se le concede actualmente, sobre todo con la creación del instrumento más conocido en la evaluación del burnout, el "Maslach Burnout Inventory" MBI- (Maslach y Jackson, 1981), instrumento al que se le ha dedicado buen número de investigaciones actuales (Rohland et al., 2004).

En nuestro país se han llevado a cabo algunos estudios que ponen de manifiesto la importancia que tiene este concepto dentro de la investigación actual en el campo del estrés laboral, sobre todo para construir instrumentos que permitan una valoración correcta de este tipo de respuesta de estrés crónico (García y Velandrino, 1992; Moreno-Jiménez, Bustos, Matallana, y Miralles, 1997; Olivares y Gil-Monte, 2007). Algunas de estas investigaciones sobre el burnout se han centrado en el colectivo de profesionales que se dedican a la enseñanza (de la Cruz y Urdiales, 1996). La evaluación del estrés entre los profesionales que ejercen su actividad en el campo de la docencia es importante porque la calidad de la educación depende en buena medida de la salud mental de los propios profesores. Las situaciones prolongadas de estrés pueden devenir en estados de agotamiento y depresión, lo cual, a su vez, puede degenerar en un estado de evitación de los problemas y de negación de los conflictos (Moriana y Herruzo, 2004).

Como contrapunto a las situaciones de estrés encontramos algunos conceptos de carácter positivo como pueden ser el engagement (Salanova, Martínez, Bresó, Llorens y Grau, 2005), la autoeficacia general o la autorregulación. Algunas investigaciones recientes han señalado la importancia de la conducta proactiva en la elaboración de modelos sobre el diseño laboral (Carver y Scheier, 1998; Fuller, Marler y Hester, 2006; Salanova, Bresó y Schaufeli, 2005).

En este estudio se han elegido dos conceptos marcados con este carácter positivo: la autoeficacia general y la autorregulación. En el primer caso, la autoeficacia general percibida está vinculada a un sentimiento optimista de competencia personal que explicaría la motivación y el logro de los seres humanos (Scholz, Gutiérrez-Doña, Sud y Schwarzer, 2002). Algunos estudios recientes han buscado el papel que la autoeficacia laboral ejerce como variable mediadora entre la calidad del trabajo y la ejecución y el estado de salud (Lubbers, Loughlin y Zweig, 2005), mientras que otros se han interesado en la aportación de la autoeficacia al bienestar a través de los objetivos personales (Sansinenea et al., 2008). Por su parte, la autorregulación puede ser considerada como una disposición variable, responsable de las acciones que facilitan la regulación personal en un abanico amplio de situaciones (Luszczynska, Diehl, Gutiérrez-Doña, Kuusinen y Schwarzer, 2004). La elección de estas dos variables como elementos de contraste viene justificada por el hecho de que la autorregulación de las emociones viene definida como la modificación de la experiencia subjetiva de las emociones, de tal modo que permita la obtención de los objetivos personales (Lawton, 2001). Parece lógico esperar que las personas que presentan baja autorregulación de las emociones negativas, como es el caso de la irritación laboral, tengan dificultades a la hora de experimentar emociones negativas, estén preocupados con frecuencia y tengan dificultades para desconectar de sus estados emocionales presentes.

Dentro de este contexto teórico, el primer objetivo que nos planteamos en este estudio es comprobar que los resultados obtenidos por los profesores en su estado de irritación, valorado mediante la Escala de Irritación (Mohr, 1986), correlacionarán de forma positiva y estadísticamente significativa con los resultados obtenidos en la valoración del estado de burnout. De este modo, se obtendrá una prueba de la validez convergente y de constructo de la Escala de Irrita- 
ción mediante su correlación con el "burnout", desde el momento en que ambos instrumentos evalúan conceptos estrechamente relacionados con la experiencia de estrés laboral.

Por contra, un segundo objetivo que este estudio plantea es verificar que se obtendrán correlaciones estadísticamente significativas pero negativas entre la irritación y constructos que estén vinculados a un afrontamiento positivo de las situaciones de estrés, en este caso la autoeficacia general y la autorregulación.

En definitiva, este trabajo se presenta como un estudio más para obtener evidencia acerca de la validez de las puntuaciones de la Escala de Irritación utilizando, en este caso, métodos basados en la relación con otras variables. Como es bien sabido, el proceso de validación de cualquier prueba es (o debería ser) un proceso continuo de acumulación de evidencias obtenidas con distintos métodos, de modo que la evidencia acumulada permita interpretar las puntuaciones de la prueba de un modo determinado o apoyar una determinada utilización de las puntuaciones para un objetivo previamente especificado. Ése es el marco de trabajo ideal con el que hay que operar y así ha sido definido en las últimas ediciones de los Standards for Educational and Psychological Measurement (1999), referente incuestionable desde hace décadas acerca de cómo proceder a la hora de medir variables psicológicas y educativas.

\section{Método}

\section{Participantes}

Para llevar a cabo esta investigación 106 profesores de Educación Primaria participaron en el estudio, todos los profesores se encontraban ejerciendo su actividad docente en provincias de la zona centro de España, concretamente en las comunidades autónomas de Madrid y de Castilla y León. El criterio de inclusión fue que los sujetos estuvieran activos en el momento de contestar a los cuestionarios. La muestra seleccionada ha sido bastante heterogénea en relación a las variables requeridas (sexo, edad, estado civil, tiempo laboral activo y nivel de estudios). Aunque la muestra no es muy amplia, es suficiente para el objetivo de un estudio exploratorio como éste (American Educational Research Association, 1999).

En cuanto a la distribución por géneros, la representación dentro de la muestra es muy desigual, siendo mayor el número de mujeres, con un total de 70 (66.04\%), frente al número de hombres, que representaban sólo un $33.96 \%$ de la muestra. Esta situación refleja justamente lo que sucede en la población docente actual, donde la proporción de mujeres es también más alta que la de varones, dada la gran feminización de la enseñanza; en este sentido, el censo docente consultado en una de las provincias donde se realizó el estudio refleja esta distribución, con un $65.7 \%$ de mujeres y un $34.3 \%$ de hombres.

Por otro lado, la distribución según la edad de los participantes fue la siguiente: con edades comprendidas entre 21 y 40 años, había un total de 46 profesores (43,40\%), y con edades entre 41 y 60 , un total de 60 participantes $(56,60 \%)$. La edad media de los participantes fue de 40 años, con una desviación típica de 10. En la muestra no había ningún profesional menor de 21 años ni mayor de 60. La muestra era bastante diversa en cuanto a la situación laboral activa, desde tan solo un año de experiencia el sujeto que menos tenía, hasta más de 41 años de ejercicio para el más veterano.

Por último, considerando el nivel de estudios, la mayoría de los participantes, 86, tenían el título de diplomado, una cifra que representa el $81,13 \%$ del total de la muestra; los 20 sujetos restantes contaban con estudios de licenciatura, esto es un $18,87 \%$ del total de la muestra seleccionada.

\section{Instrumentos}

En esta investigación se utilizaron 4 instrumentos de evaluación. En primer lugar, la escala cuya validez se pretende comprobar, la Escala de Irritación (Mohr, 1986) es un instrumento que consta de 8 ítems distribuidos en 2 dimensiones: Irritación Cognitiva (ej. ítem: Me resulta difícil desconectar después del trabajo) e Irritación Emocional (ej. ítem: Me enfado con facilidad). Los sujetos tienen que valorar sus respuestas en un formato tipo Likert con valoraciones que oscilan entre 1 (Muy en desacuerdo) y 7 (Muy de acuerdo). La primera dimensión es denominada por el equipo de la universidad de Leipzig irritación cognitiva (IC), concepto que tiene que ver con una tendencia a dar vueltas a los mismos pensamientos y pensar sobre ellos de una forma recurrente en ausencia de las demandas ambientales inmediatas que los requieran. Por otro lado, la irritación emocional (IE), llamada también irritabilidad (irritability), es un aspecto que guarda relación con una cierta disposición emocional a sentir y responder desde un estado interno de ira o incluso agresividad ante la pérdida de incentivos para conseguir un determinado propósito importante para el individuo. En investigaciones previas se han obtenido resultados consistentes en fiabilidad (.86 para la irritación emocional y .87 para la cognitiva) con muestras de docentes de educación secundaria (Merino, Carbonero, Moreno-Jiménez y Morante, 2006) y validez, apreciándose correlaciones significativas entre la irritación y otras variables como depresión $(r=.29, p<.01)$, impaciencia $(r=.70, p<.001)$ o incapacidad para relajarse $(r=.73, p<.001)$ (Mohr et al., 2006). Además, los análisis realizados con la escala parecen confirmar la presencia de una estructura de dos dimensiones dentro de la escala, un dato que ha sido validado con varias muestras ocupacionales y en diferentes países (Merino y Pedraza, 2007; Mohr et al., 2006). La escala fue traducida directamente al idioma castellano por los investigadores de la Universidad de Leipzig en Alemania, en un segundo momento, la escala fue traducida de nuevo al alemán por profesores bilingües ("back-translation").

El segundo instrumento utilizado fue el Cuestionario Breve de Burnout-CBB- (Moreno-Jiménez et al., 1997), adaptado para una muestra de profesores. Este cuestionario se valora mediante respuestas que van desde 1 (En ninguna ocasión) a 
5 (En la mayoría de las ocasiones). Se trata de un cuestionario que consta de 21 ítems organizados en tres bloques: antecedentes del burnout, factores del síndrome y consecuencias del burnout. El primer bloque, antecedentes del burnout, está configurado por tres aspectos que la teoría ha considerado relacionadas con el síndrome: características de la tarea, tedio y organización (ej. ítem: Mi trabajo actual carece de interés). El segundo componente integra los tres factores del sindrome recogidos en el modelo presentado por Maslach y Jackson (1981): cansancio o agotamiento emocional, realización personal en el trabajo y despersonalización (ej. ítem: Cuando estoy en mi trabajo me siento de malhumor). Finalmente, el tercer bloque, que integra los ítems relativos a las consecuencias del burnout, tiene en cuenta las consecuencias físicas, las consecuencias sobre el clima familiar y el rendimiento laboral (ej. ítem: El trabajo está afectando a mis relaciones familiares y personales). Las propiedades psicométricas de este instrumento son bastante consistentes, tanto en fiabilidad (valores alpha de .74) como en validez convergente $(r=.69)$, utilizando como referencia el MBI (Moreno-Jiménez et al., 1997).

El tercer instrumento utilizado fue la Escala de Autoeficacia General (General Self-Efficacy Scale-GSES-). Esta escala ofrece una puntuación global en la sensación personal de confianza en las propias capacidades para hacer frente a las demandas del ambiente en que una persona se desenvuelve (ej. ítem: Puedo resolver problemas difíciles si me esfuerzo lo suficiente). En 1981 la versión original fue reducida a los actuales 10 ítems y adaptada a 28 idiomas. La última versión de esta escala ha sido utilizada en numerosos países y arroja unos índices muy aceptables de fiabilidad, valores alpha entre .75 y .91 en las distintas investigaciones internacionales llevadas a cabo; en cuanto a la validez, se han encontrado correlaciones significativas con otras variables como optimismo $(r=.52)$ y ansiedad ( $r=-.42$ ) (Scholz et al., 2002). Este instrumento ya ha sido objeto de estudio y adaptación con muestras españolas (Baessler y Schwarzer, 1996).

Por último, el cuarto instrumento utilizado en esta investigación fue la Escala de Autorregulación (Self-Regulation Scale -SRS-), concretamente la última versión reducida a sólo 7 ítems (Luszczynska et al., 2004), (ej. ítem: Si es necesario, yo puedo concentrarme en una sola actividad por largo tiempo). Existe una versión original con 10 ítems que fue desarrollada como una parte de un proyecto de investigación sobre el estrés y las estrategias de afrontamiento entre profesores de escuela. Las versiones en español de la Escala de Autoeficacia General Percibida y de la Escala de Autorregulación fueron facilitadas directamente por el equipo de investigación de la Freie Universität de Berlín. Los valores de consistencia interna de la escala oscilan entre valores alpha de .63 y .87; en cuando a la validez se han encontrado valores significativos con la autoeficacia general percibida $(r=.75, p<.001)$ o la depresión $(r=$ $.25, p<.001)$ (Luszczynska et al., 2004).

\section{Procedimiento}

La recogida de datos tuvo lugar durante un curso escolar, durante el periodo correspondiente al segundo trimestre, es decir desde el mes de enero hasta abril. Se solicitó la colaboración voluntaria de los profesores a través de los directores de los centros. Se trata de un muestreo no probabilístico o aleatorio-accidental. Una vez confirmada la colaboración, a los profesores se les explicó personalmente el procedimiento para rellenar los cuestionarios. Cada participante se podía llevar los instrumentos para contestarlos en su casa. La tasa de respuesta obtenida fue superior al $80 \%$. Por su parte, la tasa de rechazos debidos a deficiencias fue inferior a un $10 \%$.

\section{Análisis}

Para realizar los análisis estadísticos se utilizó el paquete estadístico SPSS 14.0 para Windows, obteniendo datos correspondientes a la fiabilidad de los tres cuestionarios mediante el coeficiente alpha de Cronbach, así como de las correlaciones bivariadas entre los instrumentos mediante el coeficiente de correlación de Pearson, con prueba de significación bilateral.

\section{Resultados}

Los resultados que vamos a comentar en esta investigación están centrados fundamentalmente en la comprobación de las hipótesis planteadas más arriba en la introducción, es decir, las correlaciones que presenta la Escala de Irritación con el Cuestionario Breve de Burnout, la Escala de Autoeficacia General y la Escala de Autorregulación.

Tabla 1. Estadísticos descriptivos y valores de fiabilidad alpha de Cronbach.

\begin{tabular}{|c|c|c|c|c|c|c|}
\hline & $\mathrm{N}$ & Mínimo & Máximo & Media & d.t. & alpha \\
\hline Escala de Irritación & 106 & 8 & 52 & 29.19 & 10.17 & .89 \\
\hline Irritación Cognitiva & 106 & 26 & 86 & 12.17 & 4.499 & .86 \\
\hline Irritación Emocional & 106 & 20 & 63 & 17.02 & 7.15 & .90 \\
\hline Cuestionario de Burnout & 106 & 13 & 28 & 50.39 & 10.503 & .89 \\
\hline Factores de Burnout & 106 & 3 & 21 & 18.93 & 4.777 & .81 \\
\hline Síndrome de Burnout & 106 & 5 & 33 & 23.16 & 4.948 & .78 \\
\hline Consecuencias Burnout & 106 & 11 & 37 & 8.29 & 2.48 & .75 \\
\hline Autoeficacia General & 106 & 10 & 37 & 30.19 & 5.14 & .82 \\
\hline Autorregulación & 106 & 3 & 15 & 19.57 & 3.058 & .79 \\
\hline
\end{tabular}

En la Tabla 1 se presentan los resultados correspondientes al análisis de consistencia interna, donde se recogen los estadísticos descriptivos, incluyendo los valores máximos y mínimos para cada una de las escalas con las que se está tra- 
bajando, y los datos de fiabilidad de las variables consideradas. Para la Escala de Irritación se obtuvo un coeficiente alpha de Cronbach de .89, siendo los valores para las subescalas de .86 para la irritación cognitiva y de .90 en el caso de la irritación emocional. Por su parte, en el Cuestionario Breve de Burnout, el coeficiente alpha de Cronbach obtenido alcanzó un valor de .89 , siendo los valores de las subescalas de .81 para los factores de burnout, .78 para el síndrome de burnout y en la subescala de consecuencias de burnout se obtuvo un valor de .75. Por último, para la Escala de Autoeficacia General se obtuvo un valor de .82 y para la Escala de Autorregulación un valor de .79. Estos resultados están en línea con los obtenidos en otras investigaciones previas nacionales (Merino et al., 2006) e internacionales (Scholz et al., 2002).

En la Tabla 2 aparecen los resultados correspondientes a la distribución de las puntuaciones de cada una de las escalas utilizadas, concretamente se presentan los datos correspondientes al análisis de la prueba Kolmogorov-Smirnov para una muestra, así como la asimetría y la curtosis de las distribuciones. Según estos datos, podemos comprobar que la distribución de las puntuaciones se ajusta a la distribución normal en los cuatro instrumentos de evaluación usados en el trabajo.

Tabla 2. Distribución de las puntuaciones $(\mathrm{N}=106)$.

\begin{tabular}{lllll}
\hline & Z KS & $\begin{array}{l}\text { Sig. Asint. } \\
\text { bilateral }\end{array}$ & Asimetría Curtosis \\
\hline Escala de Irritación & .552 & .921 & .138 & -.469 \\
Cuestionario de Burnout & .931 & .351 & .807 & 1.259 \\
Autoeficacia General & 1.357 & .05 & 2.495 & 14.795 \\
Autorregulación & 1.265 & .082 & .484 & .202 \\
\hline
\end{tabular}

Por otro lado, en la Tabla 3 quedan reflejados los resultados obtenidos en el análisis de correlaciones entre la Escala de Irritación, el Cuestionario Breve de Burnout, la Escala de Autoeficacia General y la Escala de Autorregulación Podemos observar el comportamiento diferencial de las dos subescalas de la Escala de Irritación con relación a las subescalas del CBB. Así, la irritación emocional presenta correlaciones estadísticamente significativas con factores de burnout $(r=.45$, $p<.01)$, síndrome de burnout $(r=.52, p<.01)$ y consecuentes de burnout $(r=.64, p<.05)$. Sin embargo, la irritación cognitiva sólo muestra correlaciones significativas con consecuentes de burnout $(r=.54, p<.01)$, observándose una falta de correlación significativa con factores de burnout $(r=14)$ y síndrome de burnout $(r=.12)$. La correlación entre las dos subescalas de irritación también es positiva y significativa $(r=.50, p<.01)$.

En esta misma tabla, también podemos observar cómo se obtienen correlaciones negativas y significativas entre la puntuación global de la Escala de Irritación y los resultados obtenidos en Escala de Autoeficacia General $(r=-.21, p<.05)$ y entre la puntuación global de la Escala de Irritación y la Escala de Autorregulación $(r=-.29, p<.01)$. Por su parte, en la subescala correspondiente a la irritación emocional también se observa un comportamiento similar al que presenta la escala general, dado que también muestra correlaciones estadísticamente significativas y negativas con la Autoeficacia General $(r=-.25, p<.05)$ y la Autorregulación $(r=-.30$, $p<.01)$. Sin embargo, en el caso de la irritación cognitiva sólo aparecen correlaciones significativas en el caso de la Escala de Autorregulación $(r=-.19, p<.05)$, ya que con la Autoeficacia general la correlación es también negativa, pero no llega a ser significativa $(r=-.08)$, aunque se aprecia una tendencia. Por último, la correlación entre las escalas de Autoeficacia General y Autorregulación también es significativa y positiva $(r=.41, p<.01)$, tal como era de esperar.

Además de estos resultados, en la Tabla 4, se presentan también los datos correspondientes al análisis de la estructura dimensional de la Escala de Irritación, como apoyo a favor de la evidencia empírica de la validez del instrumento. Esta estructura muestra la presencia de dos factores: irritación emocional (factor I) e irritación cognitiva (factor II), precisamente los mismos factores obtenidos en otras investigaciones (Merino y Pedraza, 2007; Mohr et al., 2006).

Tabla 3. Correlaciones entre la Escala de Irritación, el Cuestionario Breve de Burnout, la Autoeficacia y la Autorregulación.

\begin{tabular}{|c|c|c|c|c|c|c|c|c|c|}
\hline & 1 & 2 & 3 & 4 & 5 & 6 & 7 & 8 & 9 \\
\hline 1 Escala de Irritación & 1.00 & & & & & & & & \\
\hline 2 Irritación Cognitiva & $.79 * *$ & 1.00 & & & & & & & \\
\hline 3 Irritación Emocional & $.92 * *$ & $.50 * *$ & 1.00 & & & & & & \\
\hline 4 Cuestionario de Burnout & $.53 * *$ & $.24^{*}$ & $.60 * *$ & 1.00 & & & & & \\
\hline 5 Factores de Burnout & $.38 * *$ & .14 & $.45^{* *}$ & $.89 * *$ & 1.00 & & & & \\
\hline 6 Síndrome de Burnout & $.42 * *$ & .12 & $.52 * *$ & $.91 * *$ & $.68^{* *}$ & 1.00 & & & \\
\hline 7 Consecuencias Burnout & $.69 * *$ & $.54 * *$ & $.64^{*}$ & $.71 * *$ & $.50 * *$ & $.53 * *$ & 1.00 & & \\
\hline 8 Autoeficacia General & $-.21 *$ & -.08 & $-.25 *$ & $-.26 * *$ & $-.23 *$ & $-.20 *$ & $-.24 *$ & 1.00 & \\
\hline 9 Autorregulación & $-.29 * *$ & $-.19 *$ & $-.30 * *$ & $-.34 * *$ & $-.30 * *$ & $-.27 * *$ & $-.35^{* *}$ & $.41 * *$ & 1.00 \\
\hline
\end{tabular}


Tabla 4. Matriz de configuración con cargas factoriales para la solución de dos factores con eigenvalue mayor a 1.

\begin{tabular}{lll}
\hline Número ítem & Factor I & Factor II \\
1 & -.13 & $\mathbf{. 9 8}$ \\
2 & .04 & $\mathbf{. 9 2}$ \\
3 & $\mathbf{. 8 7}$ & -.12 \\
4 & .22 & $\mathbf{. 6 7}$ \\
5 & .74 & .20 \\
6 & $\mathbf{. 9 5}$ & -.08 \\
7 & $\mathbf{. 8 8}$ & -.00 \\
8 & $\mathbf{. 6 8}$ & .24 \\
\hline Porcentaje de varianza explicada & 56.71 & 18.88 \\
\hline
\end{tabular}

Método de extracción: Análisis de componentes principales.

Método de rotación: Normalización Oblimin con Kaiser.

Nota. Valores $\geq .40$ en negrita

\section{Discusión}

De acuerdo a los resultados obtenidos en esta investigación podemos afirmar que, en general, se confirma la validez de constructo de la Escala de Irritación en la muestra utilizada de profesores de Educación Primaria. Haciendo un análisis detenido de los resultados, se comprueba que la Escala de Irritación global, así como la subescala correspondiente a la irritación emocional sí obtienen correlaciones positivas y estadísticamente significativas con la puntuación global del Cuestionario Breve de Burnout. Además de la puntuación global en burnout, estos mismos resultados se hacen extensibles a cada una de las tres subescalas de este cuestionario: factores de burnout, síndrome de burnout y consecuentes de burnout. Sin embargo, la subescala correspondiente a la irritación cognitiva sólo correlaciona significativamente con la puntuación global del Cuestionario Breve de Burnout, así como con la subescala correspondiente a los consecuentes del burnout, apareciendo una falta de correlación significativa con las otras dos subescalas: factores de burnout y síndrome de burnout. Estos datos plantean la necesidad de investigaciones adicionales para contrastar los resultados obtenidos.

Este resultado puede ser debido al hecho de que la irritación cognitiva tenga un comportamiento distinto a la irritación emocional. Cabe pensar que la irritación emocional tiene una mayor proximidad a la experiencia de estrés y en concreto a la situación de burnout, este dato se confirma con otro tipo de muestras (Merino et al., 2006). Por su parte, la irritación cognitiva puede que no tenga una relación tan directa con la experiencia de estrés, sobre todo cuando éste tiene connotaciones negativas para el estado de salud; al fin y al cabo, la irritación cognitiva puede que guarde una relación más directa con los recursos cognitivos que una persona pone en marcha y no tienen por qué acabar en situaciones de burnout o agotamiento. Estos resultados hacen necesaria investigación adicional con otro tipo de muestras, de tal modo que se vayan encontrando respuestas explicativas a estos interrogantes.

Por otro lado, se confirma la aparición de correlaciones negativas y estadísticamente significativas entre la irritación laboral y las otras dos escalas utilizadas en esta investigación, la Escala de Autoeficacia General Percibida y la Escala de Autorregulación. Este dato era esperado ya que la Escala de Irritación evalúa dos dimensiones de la experiencia de estrés que, de algún modo, son contrarias a las sensaciones de autoeficacia y de control de la propia conducta. Algunos investigadores han apuntado la existencia de un sentimiento generalizado de autoeficacia que hace referencia a una confianza global en las propias capacidades para hacer frente a situaciones demandantes (Schwarzer, 2001; Schwarzer y Jerusalem, 1999; Sherer y Maddux, 1982). En este sentido, las personas con un mayor nivel de autoeficacia tenderían a puntuar más bajo en dimensiones que afecten a su experiencia de estrés laboral, tal como se confirma en este estudio.

Si analizamos con más detalle estos resultados, comprobamos que la relación más significativa se produce entre la irritación emocional y los valores de la autoeficacia general y la autorregulación. Mientras tanto, en el caso de la irritación cognitiva los resultados no son tan contundentes, al igual que sucedía en el caso del burnout, concretamente encontramos que la relación entre la irritación cognitiva y la autorregulación es negativa y significativa, mientras que en el caso de su relación con la autoeficacia el resultado es negativo pero no llega a ser significativo, aunque sí se aprecia una tendencia. Este hecho, como veíamos antes, puede estar relacionado con el hecho de que la irritación cognitiva tiene un comportamiento distinto a la irritación emocional, al presentar un resultado menos negativo. De hecho, en otras investigaciones previas (Merino et al., 2006), en el caso de la irritación cognitiva siempre se obtienen resultados menos contundentes que con la irritación emocional cuando se analiza la relación con otras variables que evalúan estrés laboral. Esto implica que investigaciones futuras deberían profundizar en este comportamiento diferencial por parte de las dos dimensiones de la irritación que aparece en este estudio, lo cual permitiría avanzar en el conocimiento de la naturaleza y la relación entre las dos variables y, finalmente, en la estructura del instrumento.

En cuanto a las aplicaciones prácticas que se derivan de esta investigación, queremos destacar, en primer lugar, la utilidad práctica del instrumento, la Escala de Irritación, en el contexto de la evaluación del estrés laboral de los docentes. Este instrumento puede servir para valorar de un modo preciso las intervenciones que se lleven a cabo en materia de mejora de la salud laboral y prevención del estrés, particularmente en contextos educativos, lo cual aportaría evidencia empírica a favor de la validez externa y utilidad del cuestionario, al contrastar la sensibilidad del instrumento a los cambios o ante algún tipo de tratamiento. Además, se trata de un instrumento que puede aportar datos a nivel preventivo, ya que, el conocer el estado de irritación de los docentes puede evitar males mayores como por ejemplo estados de burnout o situaciones de depresión y ansiedad, lo cual puede influir en una mala ejecución $\mathrm{y}$, en consecuencia, una pobre calidad educativa. 
Por otro lado, también parece adecuado apuntar la posible utilización de los otros dos instrumentos mencionados en esta investigación, la Escala de Autoeficacia General y la Escala de Autorregulación, como medidas de valoración para programar y elaborar intervenciones en el campo educativo que estén relacionadas con la mejora de algunos patrones de conducta que permitan responder a las demandas laborales de un modo más eficaz. Conocer y evaluar estas variables puede tener un importante valor preventivo en el mundo de la educación, por ejemplo mediante programas que incidan en el desarrollo de este tipo de variables a nivel personal y valorar su relación con el nivel de compromiso de los profesores con el centro.

Dentro de las posibles líneas de investigación futuras, una de ellas sería la de profundizar en la validez externa y utilidad del cuestionario, cuál es el valor de predicción de la escala sobre algún criterio como por ejemplo el bienestar general o el compromiso laboral, contrastar las diferencias entre grupos que puedan tener un comportamiento diferente en irritación, etc.

\section{Referencias}

American Educational Research Association, (1999). Standards for educational and psychological testing. Washington, DC: American Educational Research Association.

Baessler, J. y Schwarzer, R. (1996). Evaluación de la autoeficacia: adaptación española de la Escala de Autoeficacia General. Ansiedad y Estrés, 2(1), 1 8.

Carver, C.S. y Scheier, M.F. (1998). On the self-regulation of behavior. New York: Cambridge University Press.

de la Cruz, M.A. y Urdiales, M.C.(1996). Estrés del profesor universitario. Ansiedad y Estrés, 7(2-3), 175-194.

Dormann, C. y Zapf, D. (2002). Social stressors at work, irritation and depressive symptoms: Accounting for unmeasured third variables in a multi-wave study. Journal of Occupational and Organizational Psychology, 75(1), 33-58.

Fuller, J.B., Marler, L.E. y Hester, K. (2006). Promoting felt responsibility for constructive change and proactive behavior: Exploring aspects of an elaborated model of work design. Journal of Organizational Behavior, 27, 1089-1120.

Freudenberger, H.J. (1974). Staff burnout. Journal of Social Issues, 30, 159-165.

García, E. (1996). Estrés, profesión docente y personalidad del docente. $A n$ siedad y Estrés, 2(2-3), 245-260.

García, M. y Velandrino, A.P. (1992). EPB: Una escala para la evaluación del burnout profesional de las organizaciones. Anales de Psicología, 8(1-2), 131-138.

Heuven, E., Bakker, A.B., Schaufeli, W.B. y Huisman, N. (2006). The role of self-efficacy in performing emotion work. Journal of Vocational Behavior, 69, 222-235.

Höge, T. (2009). When work strain transcends psychological boundaries: an inquiry into the relationship between time pressure, irritation, workfamily conflict and psychosomatic complaints. Stress and Health, 25, 4151.

Latorre, I. y Sáez, J. (2009). Análisis del burnout en profesores no universitarios de la región de Murcia (España) en función del tipo de centro docente: Público versus concertado. Anales de Psicología, 25(1), 86-92.

Lawton, M.P. (2001). Emotion in later life. Current Directions in Psychological Science, 10, 120-124.

Lazarus, R.S. y Folkman, S. (1984). Stress, appraisal, and coping. New York: Springer.

Lubbers, R., Loughlin, C. y Zweig, D. (2005). Young workers' job selfefficacy and affect: Pathways to health and performance. Journal of Vocational Behavior, 67, 199-214.
Una segunda línea de investigación sería la comprobación del posible rol modulador que puede ejercer la autoeficacia en el manejo del estrés y en la ejecución laboral, tal como se ha planteado en algunas investigaciones recientes (Heuven, Bakker, Schaufeli y Huisman, 2006). Por último, otro aspecto de interés sería la investigación sobre la relación entre las variables contempladas en este estudio, irritación y estado de burnout, y los estilos de atribución (Welbourne, Eggerth, Hartley, Andrew y Sánchez, 2007).

Como limitaciones al estudio, hay que señalar que la muestra no ha sido todo lo amplia que se deseaba, por lo que resulta recomendable, como futuras líneas de investigación, realizar estudios futuros con muestras más amplias y de otros escenarios laborales. Otras limitaciones tienen que ver con la posibilidad de haber incorporado al estudio algún dato objetivo relacionado con la irritación, como por ejemplo la proporción de bajas laborales. También hay que señalar las limitaciones derivadas de utilizar autoinformes, como la deseabilidad social por parte de los sujetos a la hora de contestar, lo cual puede distorsionar su situación real.

Luszczynska, A., Diehl, M., Gutiérrez-Doña, B., Kuusinen, P. y Schwarzer R. (2004). Measuring one component of dispositional self-regulation: Attention control in goal pursuit. Personality and Individual Differences, 37(3), 555-566

Maslach, C. (1976). Burnout. Human Behavior, 5, 16-22.

Maslach, C. (1993). Burnout: a multidimensional perspective. En W.B. Schaufeli, C. Maslach y T. Marek (Eds.), Professional burnout: Recent developments in theory and research (pp. 19-32). Washington, DC: Taylor \& Francis.

Maslach, C. y Jackson, S.E. (1981). The Maslach Burnout Inventory. Manual. (2nd ed.) Palo Alto, CA: Consulting Psychologist Press.

Merino, E., Carbonero, M.A., Moreno-Jiménez, B. y Morante, M.E. (2006). La Escala de Irritación como instrumento de evaluación del estrés laboral. Psicothema, 18(3), 419-424.

Merino, E. y Pedraza, M.A. (2007). Análisis factorial de la Escala de Irritación en profesores de educación primaria: un estudio exploratorio. Ansiedad y Estrés, 13(2-3), 133-140.

Mohr, G. (1986). Die Erfassung Psychischer Befindensbeeinträchtigungen bei Industriearbeitern. Europäische Hochschulschriften. [The assessment of mental strain of industrial workers]. Frankfurt: Lang.

Mohr, G., Müller, A., Rigotti, T., Aycan, Z. y Tschan, F. (2006). The assessment of psychological strain in work contexts: concerning the structural equivalency of nine language adaptations of the Irritation-scale. European Journal of Psychological Assessment, 22(3), 198-206.

Moreno-Jiménez, B., Bustos, R., Matallana, A. y Miralles, T. (1997). La evaluación del burnout. Problemas y alternativas. El CBB como evaluación de los elementos del proceso. Revista de psicología del trabajo y las organizaciones, 13, 185-207.

Moriana, J.A. y Herruzo, J. (2004). Estrés y burnout en profesores. International Journal of Clinical and Health Psychology, 4(3), 597-621.

Öhman, L., Bergdahl, J., Nyberg, L., y Nilsson, L.G. (2007). Longitudinal analysis of the relation between moderate long-term stress and health. Stress and Health, 23, 131-138.

Olivares, V. E. y Gil-Monte, P. (2007). Análisis de las propiedades psicométricas del "Cuestionario para la evaluación del síndrome de quemarse por el trabajo" (CESQT) en profesionales chilenos. Ansiedad y Estrés, 13(2-3), 229-240.

Peltzer, K., Mashego, T.A. y Mabeba, M. (2003). Short communication: Occupational stress and burnout among South African medical practitioners. Stress and Health, 19, 275-280. 
Rohland, B.M., Kruse, G.R. y Rohrer, J.E. (2004). Validation of a single-item measure of burnout against the Maslach Burnout Inventory among physicians. Stress and Health, 20, 75-79.

Salanova, M., Bresó, E. y Schaufeli, W.B. (2005). Hacia un modelo espiral de las creencias de eficacia en el estudio del burnout y del engagement. $A n$ siedad y Estrés, 3(2-3), 319-337.

Salanova, M., Martínez, I.M., Bresó, E., Llorens, S. y Grau, R. (2005). Bienestar psicológico en estudiantes universitarios: facilitadores y obstaculizadores del desempeño académico. Anales de Psicología, 21(1), 170-180.

Sansinenea, E., Gil de Montes, L., Agirrezabal, A., Larrañaga, M., Ortiz, G. Valencia, J.F. y Fuster, M.J. (2008). Autoconcordancia y autoeficacia en los objetivos personales: ¿Cuál es su aportación al bienestar? Anales de Psicología, 24(1), 121-128.

Scholz, U., Gutiérrez-Doña, B., Sud, S. y Schwarzer, R. (2002). Is general self-efficacy a universal construct? European Journal of Psychological Assessment, 18, 242-251.

Schwarzer, R. (2001). Social-cognitive factors in changing health-related behavior. Current Directions in Psychological Science, 10, 47-51.
Schwarzer, R. y Jerusalem, M. (Eds.). (1999). Skealen zur Erfassung von Lehrerund Schülermerkmalen. Dokumentation der Psychometrischen Verfahren im Rabmen der Wissenschaftlichen Begleitung des Modellversuchs Selbstwirksame Schulen [Scales for the Assessment of Teacher and Student Characteristics]. Berlin, Germany: Freie Universität Berlin.

Sherer, M. y Maddux, J.E. (1982). The Self-Efficacy Scale: Construction and validation. Psychological Reports, 51, 663-671.

Shirom, A. (2007). The effects of work stress on health. En M.J. Schabracq, J.A.M. Winnubst y C.L. Cooper (Eds.), The Handbook of work and health psychology (pp. 383-425) (2nd ed.). Chichester, England: John Wiley \& Sons.

Welbourne, J.L., Eggerth, D., Hartley,T.A., Andrew, M.E. y Sánchez, F. (2007). Coping strategies in the workplace: Relationships with attributional style and job satisfaction. Journal of Vocational Behavior, 70, 312325 .

(Artículo recibido: 23-06-2010, revisado: 21-12-2011, aceptado: 23-1-2012) 